\title{
Inkjet Printing of Viscous Monodisperse Microdroplets by Laser-Induced Flow Focusing
}

\author{
Paul Delrot, ${ }^{1, *}$ Miguel A. Modestino, ${ }^{1,2}$ François Gallaire, ${ }^{3}$ Demetri Psaltis, ${ }^{2}$ and Christophe Moser ${ }^{1}$ \\ ${ }^{1}$ Laboratory of Applied Photonics Devices, School of Engineering, \\ École Polytechnique Fédérale de Lausanne, CH-1015 Lausanne, Switzerland \\ ${ }^{2}$ Laboratory of Optics, School of Engineering, École Polytechnique Fédérale de Lausanne, \\ CH-1015 Lausanne, Switzerland \\ ${ }^{3}$ Laboratory of Fluid Mechanics and Instabilities, School of Engineering, \\ École Polytechnique Fédérale de Lausanne, CH-1015 Lausanne, Switzerland
}

(Received 18 May 2016; revised manuscript received 20 July 2016; published 8 August 2016)

\begin{abstract}
The on-demand generation of viscous microdroplets to print functional or biological materials remains challenging using conventional inkjet-printing methods, mainly due to aggregation and clogging issues. In an effort to overcome these limitations, we implement a jetting method to print viscous microdroplets by laser-induced shockwaves. We experimentally investigate the dependence of the jetting regimes and the droplet size on the laser-pulse energy and on the inks' physical properties. The range of printable liquids with our device is significantly extended compared to conventional inkjet printers's performances. In addition, the laser-induced flow-focusing phenomenon allows us to controllably generate viscous microdroplets up to $210 \mathrm{mPas}$ with a diameter smaller than the nozzle from which they originated $(200 \mu \mathrm{m})$. Inks containing proteins are printed without altering their functional properties, thus demonstrating that this jetting technique is potentially suitable for bioprinting.
\end{abstract}

DOI: $10.1103 /$ PhysRevApplied.6.024003

\section{INTRODUCTION}

Since the early 2000s, inkjet printing has increasingly been used in manufacturing and direct-writing processes [1]. As advanced printing applications require functional inks with complex physical properties, significant effort has been devoted to the development of drop-on-demand (DOD) methods for printing materials with applications in additive manufacturing [2], biology [3], or electronics [4]. These DOD methods and devices mainly rely on piezoelectric and thermal actuation. Their mechanism is based on the generation of a pressure pulse to eject a small amount of incompressible liquid through a nozzle, hence producing one or more microdroplets.

The use of a nozzle sets two main limitations on DOD systems. First, the choice of printable inks is typically limited to $1-15 \mathrm{mPa}$ s for thermally actuated systems or up to $100 \mathrm{mPas}$ with piezoelectric actuators [1]. Higherviscosity inks present significant challenges, as they tend to obstruct the nozzle and thus require a stronger pressure pulse to be jetted due to viscous dissipation [5]. In addition, the characteristic achievable resolution, that is to say, the droplet diameter, is approximately equal to the nozzle

\footnotetext{
*Corresponding author. paul.delrot@epfl.ch

Published by the American Physical Society under the terms of the Creative Commons Attribution 3.0 License. Further distribution of this work must maintain attribution to the author(s) and the published article's title, journal citation, and DOI.
}

diameter, which results in drop sizes on the order of $15-50 \mu \mathrm{m}$. Using a piezoelectric actuator, significant resolution improvements were demonstrated by engineering the shape and amplitude of the generated pressure pulses [6-8]. However, this resolution improvement was limited to inks of low to medium viscosity $(1-50 \mathrm{mPa} \mathrm{s})$.

Alternatively, nozzle-free approaches were reported [e.g., photoacoustic actuation [9] or laser-induced forward transfer (LIFT) [10]]. Thanks to its unconfined geometry, LIFT can be employed with a micrometric resolution for the deposition of highly viscous biological inks (1-300 $\mathrm{mPas})[3,11]$ and functional inks (> $200 \mathrm{Pas})$, such as silver nanoparticle suspensions [12]. On the other hand, LIFT remains a time-consuming process, since it requires the precoating of substrates with thin films of ink [13]. Another alternative to circumvent the limitations of conventional inkjet printing is to produce highly focused jets of liquids produced by impact [14] or laser-induced bubbles [15,16]. Recently, Tagawa et al. [15] demonstrated that, by focusing a nanosecond laser pulse on an open water-filled glass capillary, a shockwave is generated which results in a supersonic flow-focused water jet.

In this study, we experimentally implement this flow-focusing effect for the generation of low-velocity $(1-2 \mathrm{~m} / \mathrm{s})$ monodisperse microdroplets $(80-200 \mu \mathrm{m})$ of a wide range of viscous liquids (2-200 $\mathrm{mPa}$ s) with the aim of broadening the range of functional materials that can be printed using DOD systems. The printability of Newtonian and non-Newtonian solutions is studied, and we demonstrate stable and controllable satellite-free microdroplet 
production for both inviscid and highly viscous liquids. In addition, we show that our device is capable of generating monodisperse viscous droplets with a subnozzle resolution for nozzle diameters larger than $200 \mu \mathrm{m}$. Finally, we show that the technique developed in this study can be used to print highly sensitive biomaterials without loss of their functionality.

\section{MATERIALS AND METHODS}

\section{A. Experimental setup}

Microdrops are produced at room temperature by focusing a 5-ns laser pulse (Continuum, ML-II, $532 \mathrm{~nm}$ ) with a $10 \times$ microscope objective on a liquid-filled glass microcapillary [see Fig. 1(a)]. The laser pulse is split in two arms by a polarizing beam splitter, and the energy is then calibrated to a ratio of $1: 1$ between the microscope objective focal spot and the energy-meter arm thanks to a half-wave plate. The energy sent on the liquid is thus monitored with the energy meter (Thorlabs, ES111C) and adjusted using neutral-density filters and the laser's internal

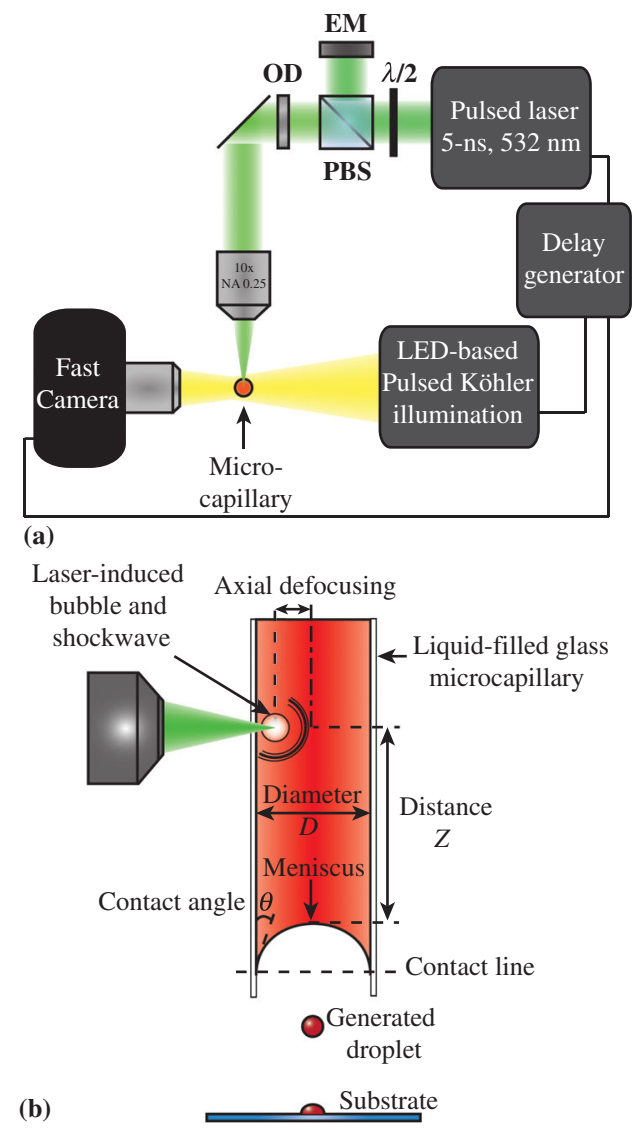

FIG. 1. Experimental setup. (a) Top view of the time-resolved imaging setup; the capillary axis is perpendicular to the graph. $\lambda / 2$, half-wave plate; PBS, polarizing beam splitter; EM, energy meter; OD, optical density. (b) Side view of the microcapillary in which the droplets are generated and a description of the experimental parameters impacting the droplet size and velocity. variable attenuator. Time-resolved imaging of the droplet generation is achieved by synchronizing a homemade LED-based pulsed Köhler illumination and a fast camera (Vision Research, Phantom Miro M310) with a delay generator (Berkeley Nucleonics, Model 577).

The glass microcapillary, which takes the role of the nozzle of the drop-on-demand device, is set in an upright configuration [see Fig. 1(b)] as in conventional inkjet printers. The Bond number $B=\Delta \rho g(D / 2)^{2} / \sigma$ [15], which represents the ratio of the gravitational and surface tension forces, is estimated to be between $10^{-4}$ and $10^{-2}$ for the different capillary sizes (where $\Delta \rho$ is the density difference between the liquid and air phases, $g$ the acceleration of gravity, $D$ the diameter of the capillary, and $\sigma$ the surface tension of the liquid). Hence, gravity is not expected to significantly affect the actuation of the liquid in the microcapillary nor the meniscus concavity. A hydrophobic treatment [17] $(1 \mathrm{H}, 1 \mathrm{H}, 2 \mathrm{H}, 2 \mathrm{H}$-perfluorooctyltrichlorosilane, ABCR, Germany) on the tip of the capillary pins the contact line of the meniscus. In this way, the contact angle $\theta$ of the meniscus and the distance $Z$ between the laser focal spot and the meniscus are controlled via gentle actuations of the syringe pump (New Era Pump Systems Inc., NE-1000) connected to the upper end of the capillary. Borosilicate glass microcapillaries with inner diameters $D$ ranging from 100 to $300 \mu \mathrm{m}$ are used (Capillary Tube Supplies Ltd. for the $100-$ and $200-\mu \mathrm{m}$ capillaries and BLAUBRAND intraMark for the $300-\mu \mathrm{m}$ capillaries). The droplets are deposited on glass microscope slides actuated by a two-axis microscope stage (Thorlabs, MLS203-1).

\section{B. Formulation of the inks}

The shear-thinning polymer precursor solutions are stained with an organic dye, oil red EGN (Aldrich, USA) at a 56- $\mathrm{m} M$ concentration to absorb laser light at $532 \mathrm{~nm}$. These solutions are 1,6-hexanediol diacrylate (HDDA; 80\%, Aldrich, USA) and trimethylolpropane triacrylate (TMPTA; > 70\%, Aldrich, USA), which were mixed with 2 wt $\%$ phenylbis(2,4,6-trimethyl-benzol)phosphine oxide (PI; 97\%, Aldrich, Germany) as a photoinitiator. SU-8 with 50\% solid content (SU-8; Gersteltec, Switzerland) is also used as received. The dynamic viscosity and surface tension of the non-Newtonian liquids are measured, respectively, with a rheometer at low shear rates (Malvern Instruments Ltd., Bohlin CVO 120) and using the pendant drop method [18].

Newtonian mixtures of water and glycerol $(99.5 \%$, Rotipuran, Roth, Germany) are studied as a model for bioinks and for comparison with the non-Newtonian behavior of the polymer precursor solutions and stained with $52 \mathrm{~m} M$ of Allura Red AC (ARAC; 80\%, Sigma Aldrich, USA). The dynamic viscosity (2-210 mPas) and density of these mixtures are estimated with Cheng's empirical formula [19], and variations of the lab temperature are monitored during the experiments. 


\section{EXPERIMENTAL RESULTS AND DISCUSSION}

\section{A. Laser-assisted microdroplet generation}

The mechanisms underlying the dynamics of droplet formation in our experiments [see Figs. 2(a) and 2(b)] correlate well to those described by Peters et al. [16] for the generation of thin supersonic jets from water-filled capillaries [15]. At time $t=0 \mu \mathrm{s}$, a laser pulse is fired and absorbed by the dyed solution, hence creating a bubble
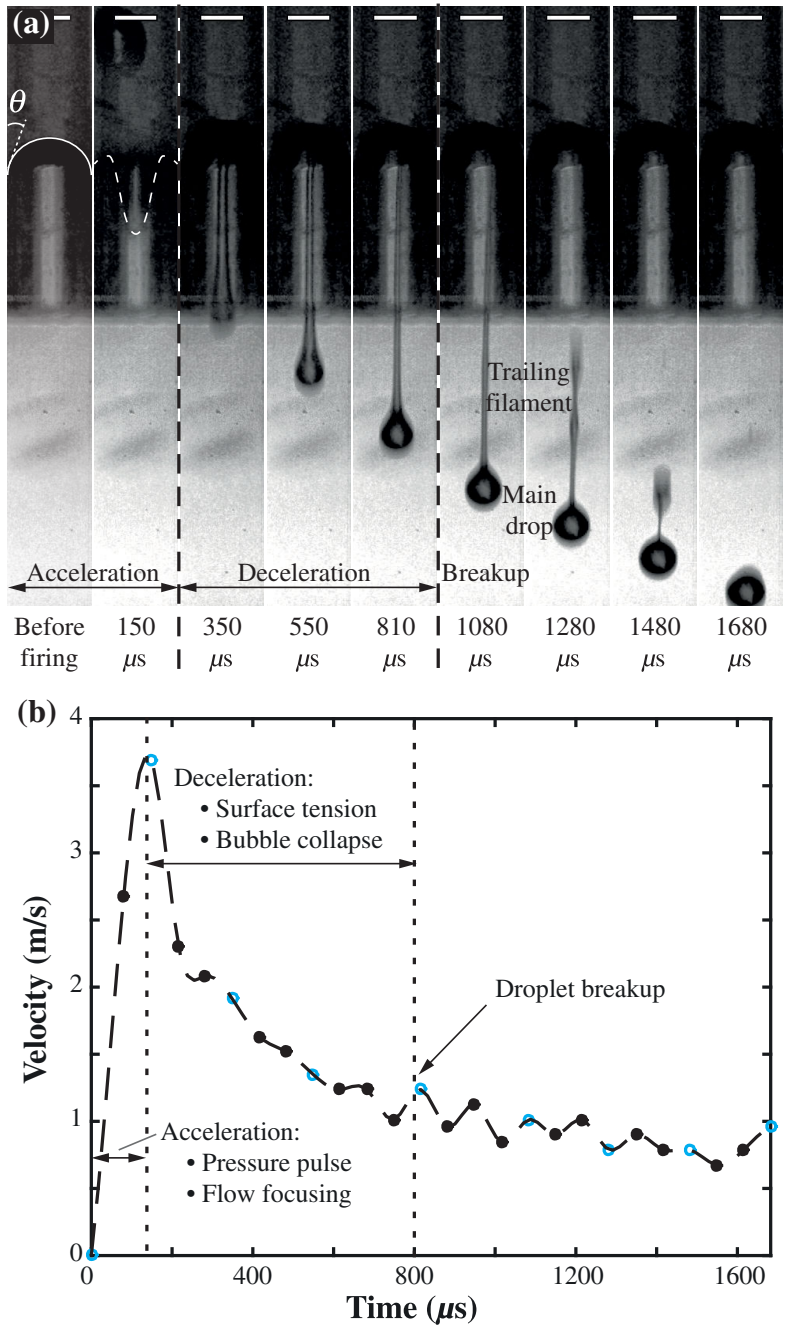

FIG. 2. (a) Series of time-resolved images of the flow-focusing phenomenon. The $300-\mu \mathrm{m}$ capillary is filled with a mixture of water and glycerol at $80 \%(\mathrm{v} / \mathrm{v})$, corresponding to a $67-\mathrm{mPa}$ viscosity at $25^{\circ} \mathrm{C}$. The liquid-air interface is highlighted with, respectively, a solid and dashed white line for clarity on the first two images corresponding to prefiring and postfiring of the laser. The contact angle of the meniscus is also highlighted. The lateral borders of the images correspond to the inner capillary walls. Distortion, due to the glass thickness of the capillary, is responsible for the black surroundings below the meniscus. Scale bar, $200 \mu \mathrm{m}$. (b) Jet dynamics corresponding to the droplet formation depicted in (a). The blue circles are the data points corresponding to the pictures in (a). The dashed line is a spline interpolation of the jet dynamics. The dotted lines delimitate the phases of jetting. [see the upper-left corner of the picture at time $t=150 \mu \mathrm{s}$ in Fig. 2(a)]. The consequent instantaneous pressure pulse first accounts for the acceleration of the liquid-air interface [see Fig. 2(b)]. The concavity of the meniscus helps to focus the flow along the axis of the capillary and results in a further acceleration of the jet. The wetting properties of the solutions on the glass capillary are demonstrated both experimentally and theoretically to be critical for the flow focusing [14-16,20], with narrow contact angles resulting in thinner jets. Therefore, in order to jet droplets as small as possible, the wetting interactions between the solutions and the capillary are controlled to assure a low contact angle. Following the acceleration phase, the bubble collapses and the fluid surface tension slows down and stretches the jet [see Figs. 2(a) and 2(b)] until it breaks up. With a correct tuning of the laser energy, the satellite-free droplet generation of viscous liquids is achieved, as shown in Fig. 2(a) (see Sec. III B for more details on the jetting regimes).

Thermal and piezoelectric actuators are able to trigger repeatable pressure pulses, which contribute to the precision of commercial inkjet printers. In order to achieve a reproducible generation of jets and droplets with our laserassisted DOD device, we design the experiments so that the laser pulse is absorbed in a well-defined focal volume of liquid from one experiment to another. Hence, the solutions are stained to absorb $90 \%$ of the laser light within a $10-\mu \mathrm{m}$ liquid thickness. In the same way, the laser focal spot is axially translated with respect to the capillary axis, to be focused in the proximity of the capillary's inner wall [see Figs. 1(b) and 2(a)]. Under these conditions, the off-axis location of the laser-induced bubble does not impact the axisymmetry of the flow focusing, provided that the distance $Z>1.5 D$ for most solutions [see Fig. 1(b)] or $Z>2 D$ for highly viscous fluids.

Another important feature of inkjet printers is their printing speed, which is typically more than $10 \mathrm{kHz}$ for conventional inkjet printers. The rates achievable by our DOD device could approach this range in the case of lowviscosity solutions, as they are limited only by the time for the meniscus to set back to its initial position after droplet generation. For a $300-\mu \mathrm{m}$ capillary, this corresponds to approximately $500 \mu \mathrm{s}$ for the least-viscous liquids to approximately $2000 \mu$ s for the most-viscous inks, as shown in Figs. 2(a) and 2(b), which would potentially result in a repetition rate between $2 \mathrm{kHz}$ and $500 \mathrm{~Hz}$. However, the effective repetition rate of our DOD system could not be investigated due to the low operating frequency of the pulsed laser $(15 \mathrm{~Hz})$.

\section{B. Jetting regimes of Newtonian and non-Newtonian inks}

The production of satellite-free microdroplets is of high interest [21-23], since satellite drops negatively impact the quality of printing. Hence, we characterize the jetting 
regimes of our laser-assisted device to ensure that a single minimal volume of liquid is dispensed at a time.

The interplay of the dynamic viscosity of the jetted inks and the laser fluence was demonstrated to be of importance in LIFT, which is a similar process to the one used in our experiments, though nozzleless. For both Newtonian [24] and non-Newtonian inks [25], viscous inks tend to stabilize the jets and allow one to select the stable printing regime on a larger range of laser fluence, thus avoiding the need for fine adjustments of the laser.

Here, with increasing laser-pulse energy we observe three different jetting regimes, which are also viscosity dependent: a subthreshold regime, a satellite-free jetting regime, and a regime with multiple droplet generation. First, at a low pulse energy, only a bubble is observed without droplet generation [see regime I in Figs. 3(a) and 3(b)]. Even if a sufficient amount of laser energy is absorbed to generate a cavitation bubble, the transferred
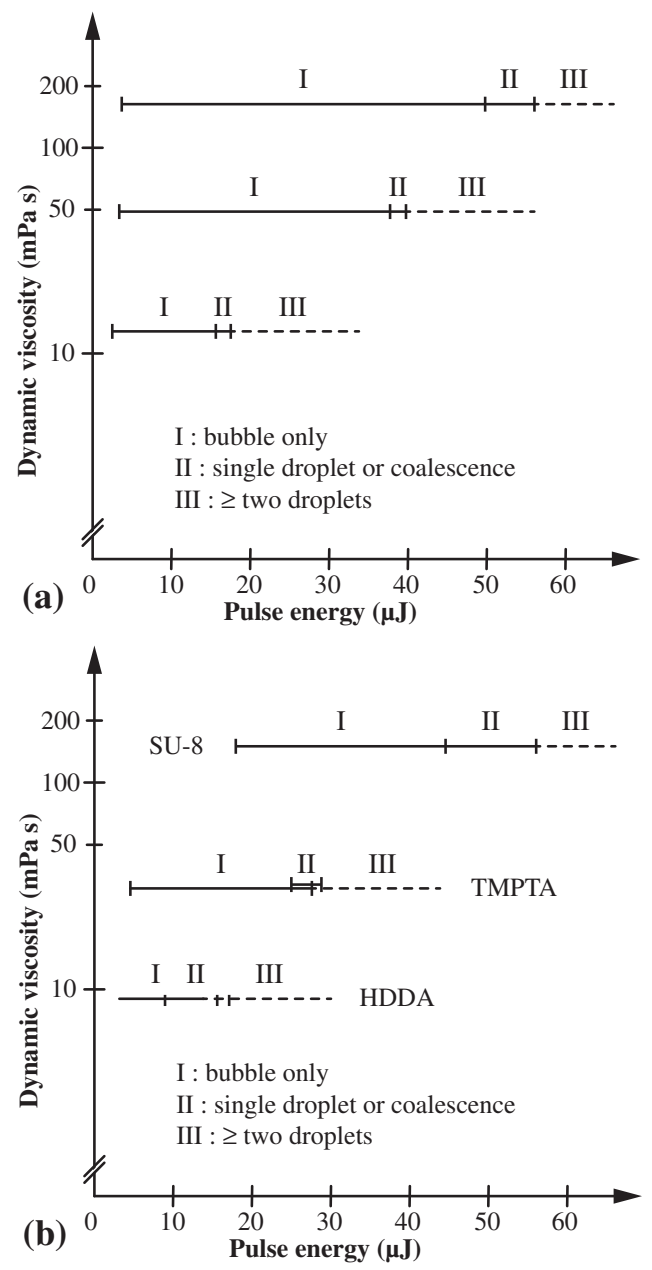

FIG. 3. Jetting regimes of inks with various dynamic viscosities for the same capillary diameter $(300 \mu \mathrm{m})$. The $y$ axis has a logarithmic scale, and the distance $Z$ between the laser focal spot and meniscus is kept constant at $500 \mu \mathrm{m}$. (a) Newtonian waterglycerol solutions. (b) Non-Newtonian polymer solutions. kinetic energy to the open interface of the liquid is insufficient to accelerate the fluid to the point of breakup. Within this regime, the surface energy and viscous dissipation prevent the formation of droplets.

By increasing the laser energy, we observe a satellite-free jetting regime with an energy threshold specific to each ink [see regime II in Figs. 3(a) and 3(b)]. At the upper energy end of this regime, two droplets can be noticed before their coalescence. Interestingly, the range of pulse energy over which stable satellite-free droplet generation is achievable increases with the dynamic viscosity of the liquids for both Newtonian and non-Newtonian inks, which is consistent with the results mentioned above for LIFT devices [24,25]. This result is likely dominated by the interplay of two competing phenomena: the axial contraction of the trailing liquid filament attached to the main drop, which favors satellite-free drop formation [see Fig. 2(a)], and the radial collapse of this filament, which on the contrary leads to the formation of satellite droplets [26]. In the case of more viscous fluids, the radial collapse will be delayed through viscous dissipation, allowing the formation of satellite-free drops even for high pulse energies that generate long trailing filaments [26].

Moreover, the velocity of the Newtonian inks at the breakup of the generated satellite-free droplets is measured to be of the order of $1-2 \mathrm{~m} / \mathrm{s}$, which is low enough to prevent the microdroplets from splashing upon the substrate [27]. On the other hand, the non-Newtonian TMPTA and SU-8 droplets have a significantly slower velocity at breakup (0.5-1.5 m/s), and drop pinch-off is also significantly delayed with respect to the Newtonian inks. Previous studies showed that polymer viscoelasticity and specifically polymer extensional strain hardening $[28,29]$ account for this phenomenon. For the same reason, we expect satellitefree droplets to be generated with more ease and, therefore, on a larger energy range using the polymer shear-thinning inks. Indeed, thanks to their elasticity, polymer inks are demonstrated [23] to be more resistant to the capillarydriven radial necking of a filament following a main drop (see, for instance, Fig. 2). However, this expected behavior cannot be clearly extrapolated from our results as seen in Figs. 3(a) and 3(b).

Finally, when a higher pulse energy is used, a third jetting regime is observed in which a faster, longer, and thinner jet is ejected and breaks up into two droplets or more [see III in Figs. 3(a) and 3(b)].

As shown in Fig. 3(b), the ejection of a TMPTA satellitefree droplet overlaps with the two other ejection regimes, which results from a poor reproducibility of the jetting process with TMPTA. Microaggregates of the light-absorbing dye are observed in the capillary, possibly due to a saturation of the ink with the dye, and we believe that the inhomogeneous distribution of the dye results in this poor reproducibility. For the other solutions, the crossover between the satellite-free regime and the other regimes 
is measured to be between $7 \%$ and $18 \%$ of the satellite-free energy range, thus leaving a sufficient working range for stable satellite-free droplet generation.

\section{Printability diagram in the satellite-free jetting regime}

Though droplet production is a complex process, the two dimensionless Reynolds and Weber numbers are commonly used to characterize inkjet printing [1,5]. The Reynolds number $(\mathrm{Re})$ represents the ratio of inertial and viscous forces. The Weber number (We) corresponds to the ratio of the kinetic and surface energies. Re and We, respectively, give an indication on whether a jet can be created and if it can lead to droplet formation. These numbers are defined as

$$
\begin{gathered}
\mathrm{Re}=\frac{\rho R V}{\eta}, \\
\mathrm{We}=\frac{\rho R V^{2}}{\sigma},
\end{gathered}
$$

where $\rho$ is the liquid density, $R$ a characteristic length, $V$ the liquid velocity, $\eta$ the liquid dynamic viscosity, and $\sigma$ its surface tension. Another dimensionless number is often used in inkjet printing to characterize the printability of a liquid without the influence of velocity [1]. The so-called Ohnesorge number $(\mathrm{Oh})$ is defined as

$$
\mathrm{Oh}=\frac{\sqrt{\mathrm{We}}}{\mathrm{Re}} .
$$

From Eqs. (1) and (2), Eq. (3) yields the following expression for $\mathrm{Oh}$ :

$$
\mathrm{Oh}=\frac{\eta}{\sqrt{\sigma \rho R}}
$$

Using these dimensionless numbers, several criteria are derived from the experimental measurements and numerical models to map the regime for stable satellite-free drop generation from a nozzle in the frame of conventional thermal and piezoelectric inkjet printers [1,5]. First, setting the characteristic length $R$ in Eq. (2) as the drop diameter, the fluid must be imparted a sufficient velocity to overcome the surface energy, yielding a criterion on We $>4$ [5], which is equivalent to the dashed line $\mathrm{Re}=2 / \mathrm{Oh}$ in Fig. 4. Moreover, in order to avoid drop splashing on the substrate, a condition on the drop terminal velocity and on the ink surface tension can be plotted as $\mathrm{OhRe}^{5 / 4}=50$ in Fig. 4 [1,5]. Finally, a previous study [30] relates the jettability of an ink through a nozzle of diameter $R$ with the Ohnesorge number Oh. If the ink is too viscous, Oh $>\sim 1$, droplet breakup cannot occur, while if $\mathrm{Oh}$ is too low, $\mathrm{Oh}<\sim 0.1$, a trail of satellite droplets is likely to be formed.

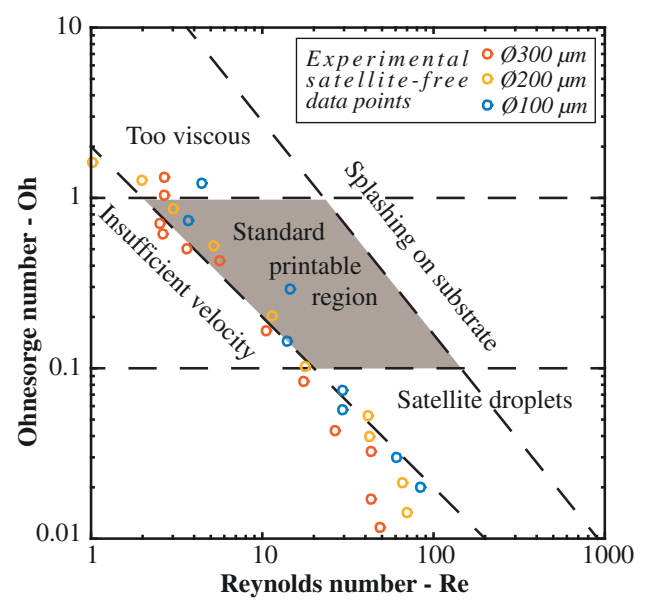

FIG. 4. Conventional inkjet-printability region adapted from Refs. [5,8] superimposed with the experimental data points obtained with our laser-assisted flow-focusing device in the satellite-free jetting regime and with the water-glycerol Newtonian mixtures. Red, yellow, and blue dots are, respectively, obtained for 300-, 200-, and 100- $\mu$ m capillary diameters. Error bars are omitted for clarity, and each data point is the results of at least ten experiments.

Based on these criteria, previous works $[1,5,8]$ showed that it is possible to build a universal printability map in Oh-Re space (see Supplemental Material Fig. S1 for a representation in Re-We space [31-33]). We adapt this diagram in Fig. 4, where the printability region for conventional inkjet printers is colored in gray. By superimposing our experimental data points for the Newtonian mixtures to this diagram, we observe that the range of Ohnesorge numbers for which satellite-free droplets are generated is extended to approximately $0.01<\mathrm{Oh}<\sim 1.5$ with our laser-assisted flow-focusing DOD system.

This result is in good agreement with a previous study in which droplets are produced from large nozzles thanks to piezoelectric-driven flow focusing [8]. The most plausible explanation for this extension of the satellite-free ejection regime with respect to conventional inkjet printers is that our device is less sensitive to the viscosity. Indeed, the measured initial velocity of the jet in the case of satellitefree droplet generation is in the range $4-7 \mathrm{~m} / \mathrm{s}$ for both Newtonian and Non-Newtonian inks and all the capillaries we test. Such initial velocities correspond to $\operatorname{Re} \sim 200$ for the least viscous inks and to $\operatorname{Re} \sim 5$ for the most viscous ones. The initial acceleration phase is then inertia dominated for the range of viscous liquids studied, which is also consistent with previous works $[14,16]$ on both inviscid and low-viscosity liquids.

Furthermore, as shown in Fig. 4, it appears that our experimental data points are roughly located along the line representing the surface energy barrier defined by Derby [5] as $\mathrm{We} \simeq 4$. This is due to the fact that we experimentally adjust the laser-pulse energy to be slightly above the droplet-generation threshold in order to obtain 


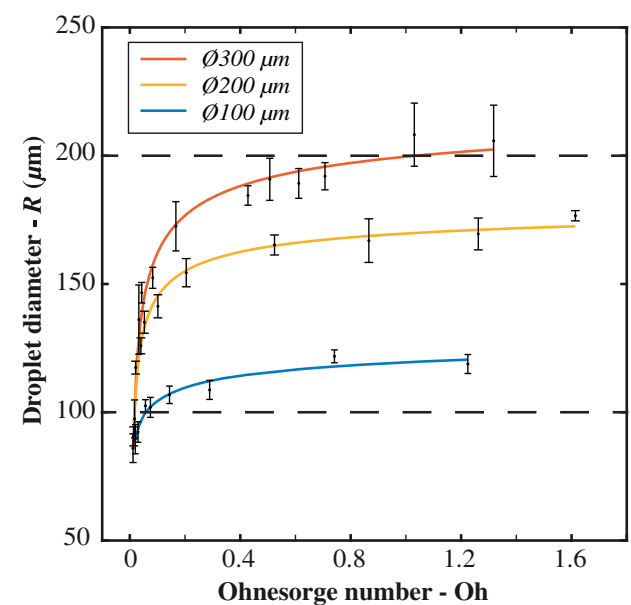

FIG. 5. Satellite-free drop diameter of water-glycerol mixtures as a function of the Ohnesorge number for different capillary sizes. The distance $Z$ between the laser focal spot and the meniscus is kept constant at $Z=500,400$, and $350 \mu \mathrm{m}$, respectively, for $D=300,200$, and $100 \mu \mathrm{m}$. Power-law fits are also displayed. Each data point is the results of at least ten experiments.

satellite-free drops. Therefore, the kinetic energy imparted to the droplets is close to the surface energy threshold, which corresponds to the criterion $\mathrm{We}>4$. The data in Fig. 4 can be further rescaled to provide a master curve for the resolution capability of our device as a function of the Ohnesorge number of the jetted Newtonian liquids (see Fig. 5). The general trend observed in Fig. 5 for all capillary sizes is a rapid increase of the droplet diameters with $\mathrm{Oh}$ for $0.01<\mathrm{Oh}<0.2$ followed by a plateau for higher Ohnesorge numbers. This plateau shows that the size of the droplets produced by our device has little dependency on the ink viscosity for highly viscous solutions. This is a promising feature, since we could possibly produce droplets of even more viscous solutions without a significant impact on the drop size.

However, experimental results suggest that the upper limit of the ejection of viscous liquids is set by the laserinduced bubbles. For highly viscous liquids, the cavitation bubbles expand along the capillary axis, and surface tension leads to the formation of static bubbles inside the capillary, thus preventing further droplet generation. A way to circumvent this issue would be to generate multiple bubbles, instantaneously or not, to create multiple pressure waves whose intensity would superimpose on the open liquid interface.

As shown in Fig. 5, satellite-free droplets with a size smaller than the nozzle from which they originate are generated up to $\mathrm{Oh}>1.3$ (corresponding to viscosities up to $210 \mathrm{mPa}$ s) for capillary diameters $D=300$ and $200 \mu \mathrm{m}$ (see Fig. 5). For a smaller orifice diameter, $D=100 \mu \mathrm{m}$, the droplets of viscosity up to $110 \mathrm{mPa}$ s $(\mathrm{Oh} \sim 1.2)$ are approximately equal to the size of the nozzle. Thus, by scaling down the orifice of our DOD device, the advantage of the subnozzle resolution is lost. A possible explanation for this result is that at a smaller nozzle scale the time for viscous effects to arise is comparable to the flow-focusing time of the device. In other words, viscous dissipation would degrade the geometry of the flow focusing. However, further theoretical studies of the phenomena can help clarify how the droplet size relates to the tube radius as well as to interpret the trend of the master curves of Fig. 5.

\section{Application to biomolecular printing}

Bioinks are highly sensitive to heat and could therefore be degraded during the laser-induced drop generation. In order to assess the potentiality of our DOD device to deliver biomolecules, bioinks containing, respectively, mouse and rabbit immunoglobulins (IgGs) are printed on poly-Llysine slides (see the Appendix for further details), and an immunoassay is performed to check the integrity of the deposited IgGs following the protocol of Ref. [34].

The droplets are generated in the satellite-free regime of ejection (see Sec. III B), and columns of rabbit IgGs are alternatively printed with columns of mouse IgGs on a slide placed $2 \mathrm{~mm}$ below the capillary tip. Prior to drying and blocking the slides with bovine serum albumin, the array is imaged under bright-field microscopy [see Fig. 6(a)]. The droplets are generated with a good reproducibility and controllability, as shown in Figs. 6(a) and 6(b), and, even when multiple droplets are generated, the axisymmetry of the jet allows us to keep a clean printing process.

Finally, after washing the slides, an immunoassay is performed with Cy3-conjugated secondary antibodies against rabbit IgG and Cy5-conjugated secondary antibodies against mouse $\operatorname{IgG}$. The fluorescent marking is specific to the mouse and rabbit IgGs [see Fig. 6(b)], thus

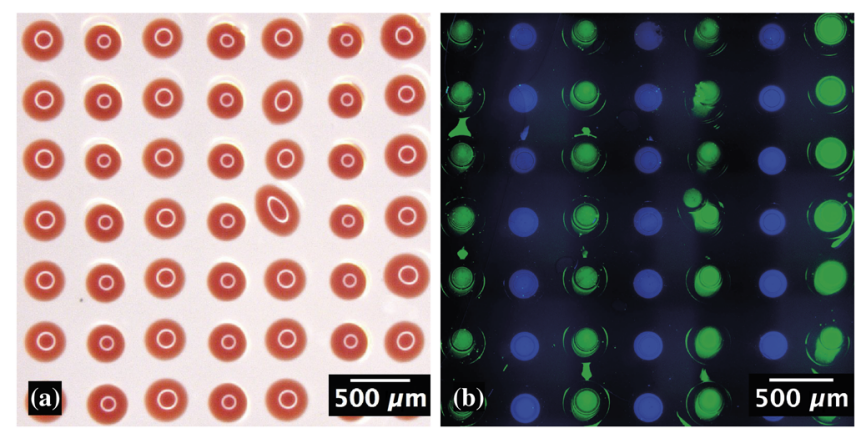

FIG. 6. (a) Array of droplets [60\% (v/v) of glycerol in PBS] with rabbit and mouse IgGs imaged with a bright-field microscope prior to droplet drying. All droplets appear red due to the light-absorbing dye. Further details on the inks' content can be found in the Appendix. (b) The same array imaged under fluorescence after drying the droplet and performing an immunoassay with fluorescent-labeled secondary anti-IgGs against rabbit (in blue) and mouse (in green) (false colors). 
demonstrating that the functionality of the IgGs is not affected by the laser-assisted printing process. This is achieved as only a small fraction of the biomaterials is exposed to the laser pulse, while the bulk of the printed material is not expected to exhibit a significant temperature increase during the process.

However, at high operating frequencies, the temperature rise in the system may become important. The role of thermal effects in the growth and collapse of a laserinduced bubble was investigated by Sun et al. [35] in a water-filled microcapillary. With similar experimental conditions to ours, they reported an abrupt rise of the bubble vapor temperature to approximately $170^{\circ} \mathrm{C}$ for a few microseconds, followed by a decrease to approximately $60{ }^{\circ} \mathrm{C}$ in approximately $400 \mu$ s. Similarly, in thermal inkjet printing, in which the fluid is also bubble actuated, inks are heated to $200-300^{\circ} \mathrm{C}$ for approximately $2 \mu \mathrm{s}$ [3], resulting in a general temperature rise of $4-10^{\circ} \mathrm{C}$ in the print head at a high frequency $(3.5 \mathrm{kHz})$ [36]. Thus, various inks with cell and protein content were reported to be viably jettable using thermal inkjet printers [3]. By analogy, our laserassisted system could potentially be viable for sensitive bioinks at high operating frequencies. This issue is not investigated here due to the low repetition rate of our laser $(15 \mathrm{~Hz})$.

\section{CONCLUSION}

Our experiments show that laser actuation within a confined geometry is capable of reproducibly generating viscous microdroplets (up to $210 \mathrm{mPa} \mathrm{s}$ ) on demand with a subnozzle resolution thanks to a flow-focusing phenomenon. A stable satellite-free drop-generation regime is evidenced for a wide range of viscous inks $(2-200 \mathrm{mPa}$ s). Selectivity of this ejection regime requires fine or coarse adjustments of the laser-pulse energy, respectively, for the inks of low and high viscosity. In addition, the proposed device allows us to extend the range of printable liquids to a range of Ohnesorge numbers $(\mathrm{Oh})$ from 0.1 to 1 for conventional inkjet printers to approximately $0.01<\mathrm{Oh}<\sim 1.5$. This extended range could impact applications in electronics and additive manufacturing for the precise printing of functional materials. The dependence of the satellite-free drop diameter on the nozzle size is experimentally investigated, and a plateau is observed at high Ohnesorge numbers, which shows that the resolution of our device would potentially not be degraded if more viscous solutions were to be printed. In addition, by successfully printing rabbit and mouse immunoglobulins, we also demonstrate that our laserassisted system has the potential to deliver bioinks. The printability of more viscous inks appears to be ultimately limited by the laser-induced bubble volume. This limitation could be overcome by generating multiple laser-induced bubbles and shockwaves using holography as a cavitation tool [37]. In the same way, replacing the microscope objective that we use as a focusing means by a multimode fiber [38,39] would allow us to guide light within the capillary and pave the way for a compact laser-assisted drop-on-demand system.

\section{ACKNOWLEDGMENTS}

P. D. thanks Dr. S. Jiguet from EPFL-LMIS4 for helpful discussions about polymer solutions. This work was supported by a grant from EPFL-STI for Additive Manufacturing.

\section{APPENDIX: IMMUNOASSAY ON THE DEPOSITED DROPLETS}

The biocompatibility of the system is assessed by a Western blot on mouse and rabbit immunoglobulin $\mathrm{G}$ ( $\mathrm{IgG}$; Sigma Aldrich, USA) following the protocol described in Ref. [34]. Specifically, the mouse and rabbit IgGs are kept at a concentration of $50 \mathrm{ng} / \mu \mathrm{l}$ in mixtures of phosphate buffered saline (PBS; 1×, Thermo Fischer Scientific, United Kingdom) and glycerol at $60 \%$ (v/v). These inks are stained with $52 \mathrm{~m} M$ of ARAC for ejection. The droplets containing IgGs are deposited on poly-L-lysine-coated slides (Sigma Aldrich, USA). Following the method in Ref. [34], the jetted droplets are dried and the slides are blocked at room temperature with $3 \%(\mathrm{w} / \mathrm{v})$ bovine serum albumin (Sigma Aldrich) in PBS for $30 \mathrm{~min}$ and then washed three times with $0.1 \%$ (v/v) Tween-20 (PBS-T; Sigma Aldrich, France) in PBS. An immunoassay is conducted by incubating $30 \mathrm{~min}$ at room temperature the slides with Cy5-conjugated secondary antibodies against mouse $\mathrm{IgG}$ (Cy-5 anti-IgG; $30 \mathrm{ng} / \mu \mathrm{L}$, Jackson Immunoresearch, United Kingdom) and Cy3-conjugated secondary antibodies against rabbit $\operatorname{IgG}(\mathrm{Cy}-3$ anti-IgG; $30 \mathrm{ng} / \mu \mathrm{L}$, Jackson Immunoresearch, United Kingdom). After that, the slides are washed three times in PBS-T, once in water, and once in $96 \%$ (v/v) ethanol. The arrays of droplets are finally imaged with a Zeiss Axioplan microscope.

[1] I. M. Hutchings and G. D. Martin, Inkjet Technology for Digital Fabrication (Wiley, New York, 2012).

[2] I. Gibson, D. Rosen, and B. Stucker, Additive Manufacturing Technologies (Springer, New York, 2015).

[3] S. V. Murphy and A. Atala, 3D bioprinting of tissues and organs, Nat. Biotechnol. 32, 773 (2014).

[4] B. J. de Gans, P. C. Duineveld, and U. S. Schubert, Inkjet printing of polymers: State of the art and future developments, Adv. Mater. 16, 203 (2004).

[5] B. Derby, Inkjet printing of functional and structural materials: Fluid property requirements, feature stability, and resolution, Annu. Rev. Mater. Res. 40, 395 (2010).

[6] A. U. Chen and O. A. Basaran, A new method for significantly reducing drop radius without reducing nozzle radius 
in drop-on-demand drop production, Phys. Fluids 14, L1 (2002).

[7] H. Y. Gan, X. Shan, T. Eriksson, B. K. Lok, and Y. C. Lam, Reduction of droplet volume by controlling actuating waveforms in inkjet printing for micro-pattern formation, J. Micromech. Microeng. 19, 055010 (2009).

[8] A. A. Castrejón-Pita, J. R. Castrejón-Pita, and G. D. Martin, A novel method to produce small droplets from large nozzles, Rev. Sci. Instrum. 83, 115105 (2012).

[9] T. Lee, H. W. Baac, J. G. Ok, H. S. Youn, and L. J. Guo, Nozzle-Free Liquid Microjetting via Homogeneous Bubble Nucleation, Phys. Rev. Applied 3, 044007 (2015).

[10] A. Piqué, R. Auyeung, J. Fitzgerald, and D. B. Chrisey, Direct-write laser transfer and processing, U.S. Patent No. 7,001,467 (2006).

[11] F. Guillemot, A. Souquet, S. Catros, B. Guillotin, J. Lopez, M. Faucon, B. Pippenger, R. Bareille, M. Rémy, S. Bellance, P. Chabassier, J. C. Fricain, and J. Amédée, High-throughput laser printing of cells and biomaterials for tissue engineering, Acta Biomater. 6, 2494 (2010).

[12] D. Munoz-Martin, C. F. Brasz, Y. Chen, M. Morales, C. B. Arnold, and C. Molpeceres, Laser-induced forward transfer of high-viscosity silver pastes, Appl. Surf. Sci. 366, 389 (2016).

[13] M. Duocastella, A. Patrascioiu, J. M. Fernández-Pradas, J. L. Morenza, and P. Serra, Film-free laser forward printing of transparent and weakly absorbing liquids, Opt. Express 18, 21815 (2010).

[14] A. Antkowiak, N. Bremond, S. Le Dizès, and E. Villermaux, Short-term dynamics of a density interface following an impact, J. Fluid Mech. 577, 241 (2007).

[15] Y. Tagawa, N. Oudalov, C. W. Visser, I. R. Peters, D. van der Meer, C. Sun, A. Prosperetti, and D. Lohse, Highly Focused Supersonic Microjets, Phys. Rev. X 2, 031002 (2012).

[16] I. R. Peters, Y. Tagawa, N. Oudalov, C. Sun, A. Prosperetti, D. Lohse, and D. van der Meer, Highly focused supersonic microjets: Numerical simulations, J. Fluid Mech. 719, 587 (2013).

[17] N. R. Glass, R. Tjeung, P. Chan, L. Y. Yeo, and J. R. Friend, Organosilane deposition for microfluidic applications, Biomicrofluidics 5, 036501 (2011).

[18] P.-G. de Gennes, F. Brochard-Wyart, and D. Quéré, in Capillarity and Wetting Phenomena (Springer New York, New York, 2004), pp. 33-67.

[19] N.-S. Cheng, Formula for the viscosity of a glycerol-water mixture, Ind. Eng. Chem. Res. 47, 3285 (2008).

[20] R. Bergmann, E. de Jong, J.-B. Choimet, D. van der Meer, and D. Lohse, The origin of the tubular jet, J. Fluid Mech. 600, 19 (2008).

[21] F. G. Tseng, C. J. Kim, and C. M. Ho, A high-resolution high-frequency monolithic top-shooting microinjector free of satellite drops-Part I: Concept, design, and model, J. Microelectromech. Syst. 11, 427 (2002).

[22] S. D. Hoath, S. Jung, W.-K. Hsiao, and I. M. Hutchings, How PEDOT:PSS solutions produce satellite-free inkjets, Org. Electron. 13, 3259 (2012).
[23] H. J. Shore and G. M. Harrison, The effect of added polymers on the formation of drops ejected from a nozzle, Phys. Fluids 17, 033104 (2005).

[24] V. Dinca, A. Patrascioiu, J. M. Fernández-Pradas, J. L. Morenza, and P. Serra, Influence of solution properties in the laser forward transfer of liquids, Appl. Surf. Sci. 258, 9379 (2012).

[25] M. Gruene, C. Unger, L. Koch, A. Deiwick, and B. Chichkov, Dispensing pico to nanolitre of a natural hydrogel by laser-assisted bioprinting, Biomedical engineering 10, 19 (2011).

[26] S. D. Hoath, S. Jung, and I. M. Hutchings, A simple criterion for filament break-up in drop-on-demand inkjet printing, Phys. Fluids 25, 021701 (2013).

[27] E. R. Lee, Microdrop Generation (CRC Press, Boca Raton, FL, 2002).

[28] O. A. Basaran, H. Gao, and P. P. Bhat, Nonstandard inkjets, Annu. Rev. Fluid Mech. 45, 85 (2013).

[29] Y. Amarouchene, D. Bonn, J. Meunier, and H. Kellay, Inhibition of the Finite-Time Singularity during Droplet Fission of a Polymeric Fluid, Phys. Rev. Lett. 86, 3558 (2001).

[30] D. Jang, D. Kim, and J. Moon, Influence of fluid physical properties on ink-jet printability, Langmuir 25, 2629 (2009).

[31] C. Clanet, C. Béguin, D. Richard, and D. Quéré, Maximal deformation of an impacting drop, J. Fluid Mech. 517, 199 (2004).

[32] C. W. Visser, P. E. Frommhold, S. Wildeman, R. Mettin, D. Lohse, and C. Sun, Dynamics of high-speed micro-drop impact: numerical simulations and experiments at frame-toframe times below 100 ns, Soft Matter 11, 1708 (2015).

[33] See Supplemental Material at http://link.aps.org/ supplemental/10.1103/PhysRevApplied.6.024003 for a representation of the experimental data points in Re-We space.

[34] M. Duocastella, J. M. Fernández-Pradas, J. L. Morenza, D. Zafra, and P. Serra, Novel laser printing technique for miniaturized biosensors preparation, Sens. Actuators B 145, 596 (2010).

[35] C. Sun, E. Can, R. Dijkink, D. Lohse, and A. Prosperetti, Growth and collapse of a vapour bubble in a microtube: The role of thermal effects, J. Fluid Mech. 632, 5 (2009).

[36] X. Cui, D. Dean, Z. M. Ruggeri, and T. Boland, Cell damage evaluation of thermal inkjet printed Chinese hamster ovary cells, Biotechnol. Bioeng. 106, 963 (2010).

[37] P. A. Quinto-Su, V. Venugopalan, and C. D. Ohl, Generation of laser-induced cavitation bubbles with a digital hologram, Opt. Express 16, 18964 (2008).

[38] I. N. Papadopoulos, S. Farahi, C. Moser, and D. Psaltis, Focusing and scanning light through a multimode optical fiber using digital phase conjugation, Opt. Express 20, 10583 (2012).

[39] D. Loterie, S. Farahi, I. Papadopoulos, A. Goy, D. Psaltis, and C. Moser, Digital confocal microscopy through a multimode fiber, Opt. Express 23, 23845 (2015). 